\title{
General Practice Services in England During the COVID-19 Pandemic and Beyond: Patient Access and Barriers
}

\author{
Author: \\ Pablo Millares Martin \\ Whitehall Surgery, Leeds, UK \\ *Correspondence to pablo.martin@nhs.net \\ Disclosure: $\quad$ The author has declared no conflicts of interest. \\ Acknowledgements: The author would like to thank Dr H. Devitt, A. Nelson, Dr S. Matharu, M. Dalton, J. \\ Woods, and L. Durno for their contributions to gaining the survey data. \\ Received: $\quad 29.07 .20$ \\ Accepted: $\quad 05.10 .20$ \\ Keywords: $\quad$ Coronavirus disease (COVID-19), general practice, health services accessibility, remote \\ consultation, technological innovations.
}

Citation:

EMJ Innov. 2021;5[1]:70-77.

\section{Abstract}

Background: During the coronavirus disease (COVID-19) pandemic, primary care services have been forced to operate differently, limiting face-to-face consultations and relying on telemedicine. This has impacted the care received by patients in need of primary care. The aim of this article was to assess the patient needs during the pandemic, their perspectives on current interactions with primary care, and the readiness for change in operating general practices in the future.

Method: A survey was conducted among patients in Leeds, UK, that explored whether patients had health needs during the pandemic, the decisions that were then taken if so, their use of online information and resources, and their satisfaction with primary care website portals and consultations.

Results: Over $75 \%$ of patients gathered information online before deciding to consult. The main effect of the pandemic was that among those whose health needs remained, 37\% did not consult, preferring to wait to see if their symptoms resolved by themselves. There was a significant statistical difference depending on age groups: among those patients aged $<30$ years, $48 \%$ did not consult a primary care physician.

Conclusion: The primary care response during the pandemic led to a large number of patients to withhold their concerns, and careful consideration is needed to access how to improve accessibility in future crises.

\section{INTRODUCTION}

The coronavirus disease (COVID-19) pandemic has changed the way general practitioners (GP) are assessing patients, as they have avoided face-to-face consultations and instead used telephone calls, video calls, and other digital solutions.' This situation has forced clinicians to work differently and patients to receive care in a limited way, breaking with the traditional, person-to-person interaction. 
The city of Leeds, with a population of around 870,000 people, is served by 94 GP practices. In the current National Health Service (NHS) structure, the practices are expected to work together serving natural communities of about 30,000-50,000 patients in what are called Primary Care Networks (PCN). In Leeds, there are $19 \mathrm{PCN}$, and among them is the Bramley, Wortley, and Middleton (BWM) PCN. The population served by this PCN numbered at around 30,000 and had slightly more children and fewer elderly citizens. The majority of these patients lived in the second most deprived area of Leeds, and a large number are in the most deprived area. The majority of patients were considered white British and the levels of long-term conditions (chronic obstructive pulmonary disease, asthma, heart disease, and minor and severe mental illness) were above the Leeds average.

To provide a better service, the BWM PCN discussed the use of a survey to gather patients' concerns on the available accessing services. The aim was to assess concerns as to whether patients were accessing services when needed, as well as whether the current process was affecting trust in primary care and if this was an indication of a permanent change in the way consultations in the future would occur. It was considered not only the responsibility of clinicians to asses whether or not to continue with any changes introduced during the crisis; ${ }^{2}$ it was deemed a coproductive effort, needed to reshape practices to make them stronger after the pandemic.

Assessing the patients' decisions in the lead up to booking a consultation, as well as gathering information on their own health, was the first step. It was accepted that many patients access the internet for health information before a GP consultation. ${ }^{3}$ It is also known that a proportion of patients in the UK access their own electronic clinical records as a result of the strong governmental push to provide digital access to patients, ${ }^{4}$ and this could be facilitated by using the same clinical software as their GP (all the practices use systmOne/SystmOnline), ${ }^{5}$ or by using the NHS app. ${ }^{6}$

Assessing consultation needs and types was needed next. The push to replace face-to-face consultations over the last few years has not been very successful, ${ }^{7}$ but it has become an urgent necessity as a consequence of the COVID-19 pandemic. Telephone and video consultations could easily replace and be more convenient than face-to-face consultations for conditions that do not require physical examination. ${ }^{8}$ However, the following questions needed to be answered: would patients agree to virtual consultations? Would they expect more examinations? Would they be less satisfied with remote consultations?

\section{METHOD}

The survey was created in Google forms and designed to follow a possible patient journey in primary care, starting with the need to satisfy a healthcare need, finding information online ("Would you look for information online before booking an appointment?"; "Have you used our website and its links to other NHS sources of information?"), deciding to seek attention or not ("Did you get the answers you were looking for? What did you do then?"), and the format of the service they interacted with ("What type of consultation have you had during the lockdown?"; “Did you feel safe coming to the practice?”).

The survey was designed to be completed in one sitting and in a short time, and there were two types of questions: closed questions (e.g., the percentage of patients looking for information online before a consultation or preferring not to consult when a symptom or health need was present), allowing for the measuring of quantitative differences in responses; and open questions ("If you had symptoms and needed help, what prevented you from contacting NHS services?"), as this allowed a more in-depth assessment of the situation, to find themes representing the behaviours, concerns, and barriers patients perceived.

The invitation was sent via SMS to patients aged $>18$ years with a mobile phone number on file. Clinical software allowed for the creation of reports to identify candidates with the inclusion criteria and to send the agreed SMS. The survey was also available on the practices' websites and posted on their social media channels. Data were collected between the $7^{\text {th }}$ of May 2020 and the $5^{\text {th }}$ of June 2020.

A mandatory question at the end of the survey asked if the patient's responses could be used 
for research purposes. Only data from patients who had agreed to this secondary purpose were included in this paper.

\section{RESULTS}

The survey analysed access to online information before consultations, decisions made after information gathering, and types of consultations and satisfaction, as well as how future primary care pathways and interactions could change. Although the invitation was sent to around 10,000 patients, there were only 1,246 responses. However, the number of responses still allowed for a valuable analysis.

This paper focusses on the findings among those who gave consent for the use of their data for research purposes (1,183 patients, $94.9 \%$ of the total); there is a variation in response numbers depending on the journey taken by the patient (Figure 1) and whether they answered all the questioned presented.

Descriptive statistical analysis indicated sex (male: 34.2\%; female: 65.3\%; 'other': $0.2 \%$; and 'prefer not to say': 0.3\%), age group (aged <30 years: 12.0\%; aged 30-65 years: 69.9.\%; and aged $>65$ years: $18.1 \%)$. It should be noted these values do not represent the population served, but simply those who responded to the survey. Respondents were also asked if they were told to 'shield' during the pandemic ('no': 76.3\%; 'yes': $11.5 \%$; and 'maybe': 12.2\%); shielding was a new concept for patients that followed guidelines introduced by the UK government in March 2020 in preparation for the country's 'lockdown', the term used to describe the requirement for people to stay at home and avoid outdoors activities. These guidelines have been regularly updated since their initial introduction. ${ }^{9}$

\section{Access to Online Information}

Not all patients accessed their records, even though they were available to them; in this study's sample, 501 patients (42.3\%) accessed their own clinical records, mainly using the practice's clinical software (420 patients, 35.5\%), while 23 (1.9\%) used the NHS app and 58 (4.9\%) used both software available to them. 903 patients (76.3\%) accessed online health information before deciding to request a consultation, while $280(23.7 \%)$ did not.

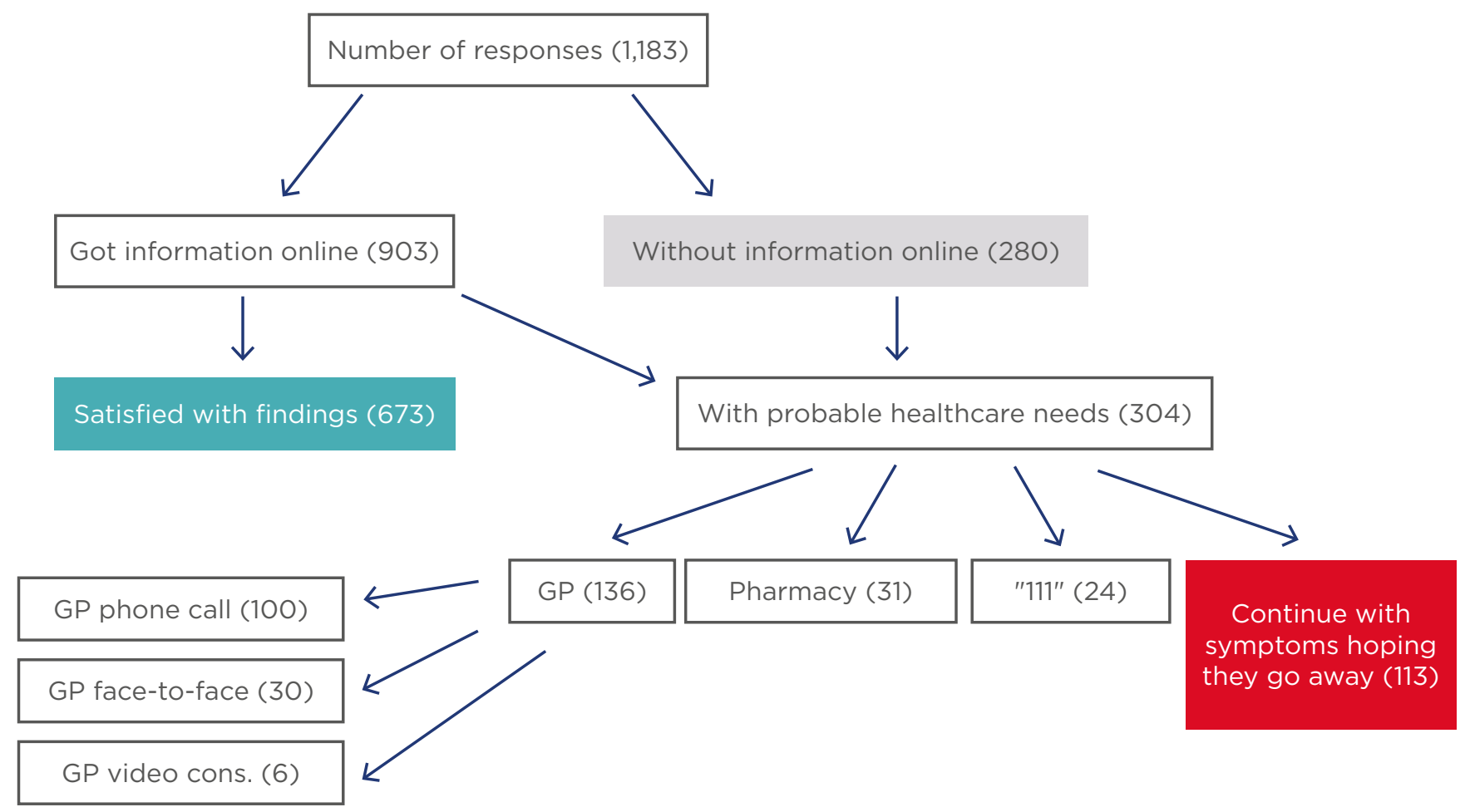

Figure 1: Flow of patients through the survey. 
Regarding the use of the primary care practices' websites to search for information, among the 848 respondents, 384 patients (45.3\%) reported using it.

Pearson chi square test of independent analysis indicated that the relationship between age groups and accessing information online was significant ( $\left.X^{2} \quad[2, n=1,183]=54.6 ; \quad p<0.001\right)$, confirming that older patients used the internet less.

Among the 369 responses to the question "Is there anything we need to change on the website?", 276 patients considered it to be 'okay' or did not make any suggestions. The following themes were identified among the 71 participants who considered changes were needed:

> Access: patients were eager to have more of their information available online (e.g., "blood test results from specialists" and "full test results"), but also wanted easier access to online forms and to the clinical software itself. There were concerns about difficulties in resetting passwords for the clinical software and the lack of a "facility to register other members of the household."

> Appointments: participants wanted an easier to book, clearer system, "to see previous appointments and be able to filter by available slots and by doctor," with more appointments available online.

> Functionality: patients asked for access to a messaging service with doctors/healthcare professionals for nonurgent needs and the ability to request sick notes and prescriptions on the same site.

> Information: requests were made for more updates and more details on specific conditions such as diabetes and Ehler-Danhlos Syndrome, because links to NHS pages did not seem to provide the desired information for them to decide when to call for an appointments.

> Navigation: Nine respondents did not like the websites, considering them "messy," "too long winded at times," "hard to follow," and needing to be easier to use for the older generation, with larger print and more clarity.

Among other resources used and the reasons for doing so, 402 respondents either did not use other sources (189 cases), 90 used NHS websites, 49 used Google, and several others used specific online providers such as medical sites (PubMed, The Royal College of General Practitioners [RCGP], WebMD), support sites (Mumsnet, Asthma UK), and UK government websites. A minority also reported asking family, pharmacists, or colleagues at work for advice. The reasons for searching online were not solely to find information, but to also obtain clarity, to get "more detailed information" before "bothering the GP," and "to prevent doctor's appointment."

The information online was treated with caution, as observed by one respondent: "The internet isn't always a good tool as some things can be so similar that you self-diagnose incorrectly as sometimes you can be met with five possible answers."

The final outcome, considered by the 903 respondents who consulted the internet, was that the healthcare need was resolved in $74.5 \%$ of cases, while $25.5 \%$ felt they still needed some help.

\section{Unresolved Healthcare Needs}

When asked what action was taken regarding the patient's needs once they had consulted the internet to provide clarify on their symptoms, among the 304 respondents, 136 patients (44.7\%) booked an appointment with a primary care physician but 113 patients (37.2\%) opted to continue with their symptoms, in the hope that they would go away. Going to the pharmacy was an option considered by 31 patients (10.2\%), while 24 (7.9\%) phoned NHS 111 number (provides urgent health advice out of hours when GP practices are closed) (Figure 2).

For statistical analysis, the actions taken by the three age groups were condensed into three options: booking an appointment, continuing with symptoms, and other support (including phoning 111 or going to pharmacy). The Pearson Chi square test of independence was then performed. There was a significant association between age group and the action taken in this patient sample ( $\left.X^{2}[4, n=304]=27 ; p<0.001\right)$.

Older patients were more likely to book an appointment rather than leave symptoms alone (66\%), while younger patients would do the opposite ( $37 \%$ for those aged $<30$ years old, and $35 \%$ for those aged 30-65 years) (See Table 1). 


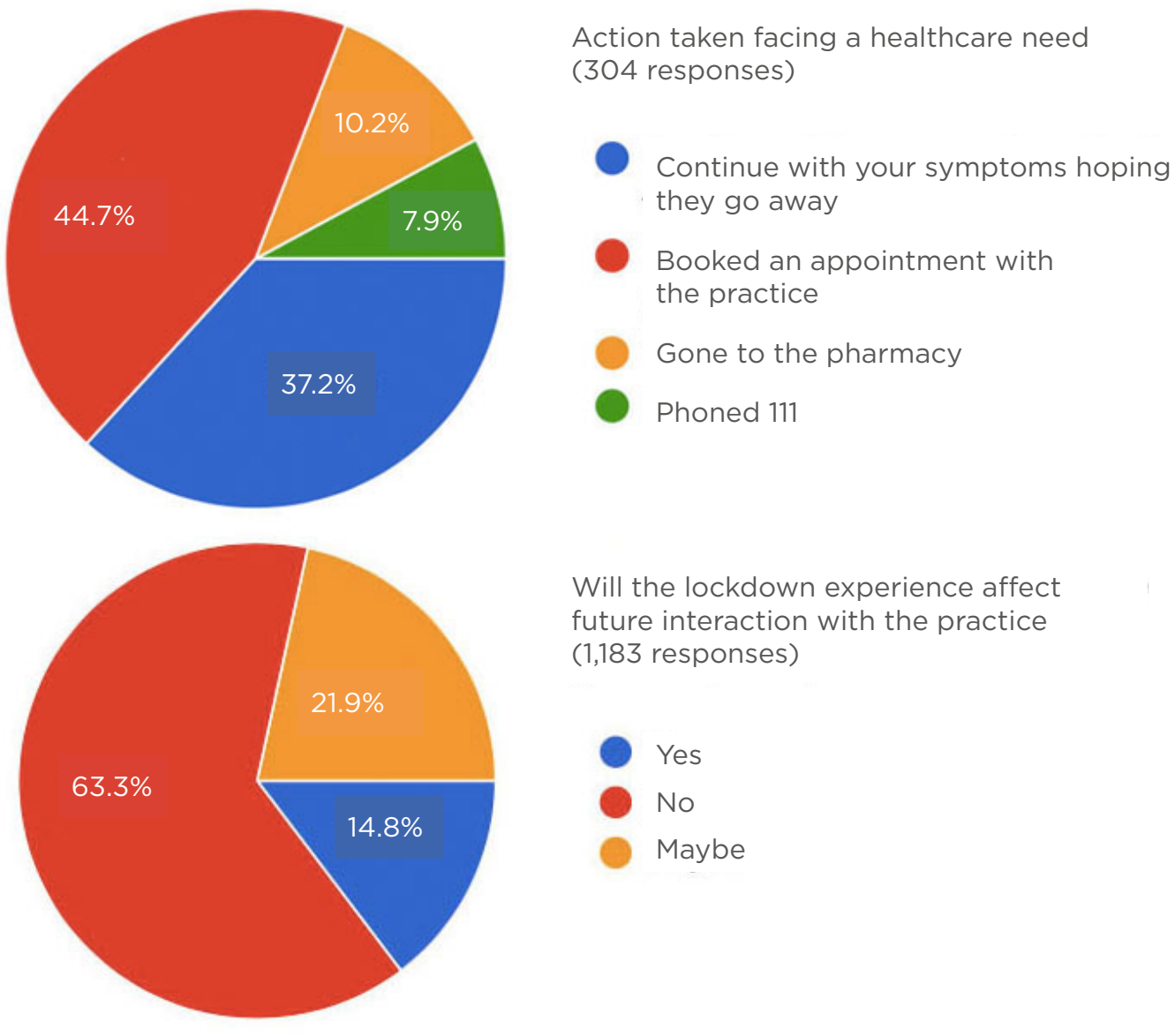

Figure 2: Visual representation of actions taken during the coronavirus disease pandemic and views on future interaction with primary care.

When considering the statistical analysis of those who were shielding, the actions were condensed into two groups depending on whether the individual sought medical attention or not. The Pearson Chi square test of independence was subsequently performed (See Table 1). The relationship between these two variables was significant $\left(X^{2}[2, n=304]=6.3 ; p=0.0042\right)$, although it was weaker than the age-related association. Patients who were uncertain about their shielding status were more likely to seek help (73\%), compared to those who were shielding (58\%) and those not shielding (62\%).

When patients were asked "If you had symptoms, what prevented you from contacting NHS services?" the themes identified were:

> Access limitations: an inability to book appointments online or to get an appointment soon enough, and several responded that "anything that would need outside referral has been put on hold/telephone only," or "I want to see somebody, not talk over the phone."
> Burden: comments such as "I don't want to burden the NHS," "I feel bad imposing on NHS at this time," or "the NHS have a lot going on at the moment," were made.

> COVID-19: whether the patients suffered from it, at higher risk, or simply concerned about contracting it, prevented patients to contact services.

\section{Consultations During Lockdown}

The UK Government adopted the slogan "Stay at Home, Protect the NHS, Save Lives", which triggered a belief among $34.5 \%$ of patients that primary care was not open as usual. Additionally, among the 447 respondents, $15.7 \%$ of individuals considered that NHS 111 and pharmacies were not offering their regular services.

Patients during the lockdown had been offered telephone calls or video consultations as their first-line of contact in the primary care setting and, when required, face-to-face followup consultations ensued. Among the survey 
respondents, 136 patients had a consultation; 100 (73.5\%) had a telephone call, 30 (22.1\%) had a face-to-face discussion, and six (4.4\%) had a video consultation. Among the 30 patients that came to the practice, 28 (93.3\%) felt safe coming, while two (6.9\%), when asked if they would feel safe, responded "maybe."

When patients were asked if the type of consultation received was sufficient to assess their needs, 27 of the 30 that were seen faceto-face agreed (90\%) and three responded with "maybe." In contrast, among the 100 patients who had telephone consultations, 79 (79\%) agreed that it was sufficient, nine responded "maybe," and $12(12 \%)$ believed it was not sufficient.

\section{PRIMARY CARE IN THE FUTURE}

When people were asked if the lockdown experience would affect the way they interact with their primary care practice in the future, 749 patients (63.3\%) considered it would not, 259 (21.9\%) thought it might, and 175 (14.8\%) reflected that they believed it would (Figure 2 ).
When given the option to comment, the following themes were noted:

> Avoidance: "I am worried about using up GP time," "I am nervous regarding doctors waiting rooms and other patients," and "I come in to the practice less" or "only going when I absolutely have to."

> Business as usual: "go back to using them as normal as face-to-face meetings are more effective," "I think doctors need to see you face-to-face for mental health problems," and "I prefer to speak to a person."

> COVID-19-related: being more "alert," "aware," "more cautious of keeping distance from people," and avoiding "going into the surgery where possible." These issues will probably return to normal once the pandemic is over.

> Digital changes: more online access, more video consultations and electronic communications ("I liked that I could send a picture"), and more website use.

> Pharmacy use: "I will use the chemist more."

> Telephone appointments: most believed it was likely they would be used more often;

Table 1: Action taken by patients depending on age group and shielding status.

\begin{tabular}{|c|c|c|c|c|}
\hline & Action taken (\%) & & & \\
\hline Age group & $\begin{array}{l}\text { Booked an } \\
\text { appointment with the } \\
\text { practice }\end{array}$ & $\begin{array}{l}\text { Waited for symptoms } \\
\text { to improve }\end{array}$ & $\begin{array}{l}\text { Other support (NHS } \\
111, \text { pharmacy) }\end{array}$ & Total \\
\hline$<30$ years old & $10(37 \%)$ & $13(48 \%)$ & $4(15 \%)$ & 27 \\
\hline $\begin{array}{l}\text { Between } 30-65 \text { years } \\
\text { of age }\end{array}$ & $63(35 \%)$ & $80(44 \%)$ & 39 (21\%) & 182 \\
\hline$>65$ years old & $63(66 \%)$ & $20(21 \%)$ & $12(18 \%)$ & 95 \\
\hline Total & 136 & 113 & 55 & 304 \\
\hline Shielding status & Sought help & $\begin{array}{l}\text { Waited for symptoms } \\
\text { to improve }\end{array}$ & & Total \\
\hline Maybe & $33(73 \%)$ & $12(27 \%)$ & & 45 \\
\hline No & $132(62 \%)$ & $82(38 \%)$ & & 214 \\
\hline Yes & $26(58 \%)$ & $19(42 \%)$ & & 45 \\
\hline Total & 167 & 113 & & 304 \\
\hline
\end{tabular}

NHS: National Health Service. 
"Maybe telephone consultations will become more prevalent rather than face-to-face appointments?"

Also of note, one participant commented: "I do not use computers and I have not got one. I struggle with the new phones also; this is the first time I have managed something like this," as a reflection on digital literacy.

\section{DISCUSSION}

\section{Summary}

This survey has demonstrated that patients have not been addressing their health needs during the pandemic appropriately, as a considerable proportion refrained from seeking attention when it might have been needed. There was a statistical difference in behaviour between younger individuals, who were more likely to wait for symptoms to improve, compared with older patients, who were more likely to consult. It could not be attributed to younger people accessing more online health information. Another area of probable confusion was the shielding status, and that individuals who were uncertain on their shielding status were more likely to seek attention.

Though clinicians are making decisions about changing consultation formats in the future, the patients in this study believed that long-term access to primary care would not be affected by the pandemic, and that telephone calls were generally rated as less effective at solving their health needs. There was certain resistance to moving from face-to-face consultations, despite the participants also being interested in using digital tools, if possible. If telephone consultations were to become the norm, many patients would probably oppose.

\section{Strengths and Limitations}

The survey data from this small sample obtained important information to consider regarding who is statistically more likely to seek help (patients with unclear shielding status, older patients) and the fact that a large proportion of patients appear to consult the internet before deciding to approach primary care.
The invitation to the survey by SMS limited the type of patients able to access it, although it may have focussed on the type of patients most likely to use telemedicine. This is suggestive of health inequality increasing if views of those who are not digital literate are not taken into account. The PCN population, with a considerable level of deprivation, may have reduced smartphone use compared to other communities. The survey also has limitations in that open questions were used, which tend to encourage short answers. ${ }^{10}$ It should also be noted that more female patients took part in the survey, which may be reflective of females tending to interact more with general practice; for example, in the study by Wang et al., ${ }^{11}$ the crude consultation rate was $32 \%$ lower in males than females.

\section{Comparison with Existing Literature}

This study confirmed that patient's access to online services decreases with age, ${ }^{12}$ but the amount of healthcare need that was solved by access to online information and the proportion of patients who preferred to wait with their symptoms are both areas that have not been explored before, and are quite relevant in the current context. Concerns have been raised on the impact of not seeking medical attention for symptoms, which could lead to poorer outcomes in long-term conditions like cancer, ${ }^{13}$ and the inequalities created by the shift towards telemedicine. ${ }^{14}$ In the past, changes in consultation methods came from different pressures, and innovations like electronic consultations have had less impact than expected. ${ }^{15}$ The current reality is an effect felt everywhere, and this burden needs to be assessed regularly at a national and local level. ${ }^{16}$ The survey presented focussed on general access to services, and the learning from the results could help similar processes elsewhere. Other surveys have focussed on different aspects, such as the perception of digital healthcare, ${ }^{17}$ the concerns of contracting COVID-19, ${ }^{18}$ or the preparedness of primary care..$^{19}$ Together a clear picture is emerging of the pandemic's impact on all levels of healthcare.

\section{CONCLUSIONS}

Primary care, alongside many other health services, will undergo multiple changes following the current pandemic. Understanding 
patients' perspectives, as well as clinicians' attitudes, could help to build a resilient and satisfactory consultation pathway. The impact of service digitalisation on patients who are not electronically literate needs to be explored further.

\section{Ethical Approval Statement}

Patients were asked at the end of the questionnaire if responses could be used for research purposes. Only those survey answers where the patient agreed for this secondary purpose were used.

\section{References}

1. Ohannessian R et al. Globa telemedicine implementation and integration within health systems to fight the COVID-19 pandemic: a cal to action. JMIR Public Health Surveill. 2020;6(2):e18810

2. Marshall M et al. COVID-19: a danger and an opportunity for the future of general practice. $\mathrm{Br} J$ Gen Pract. 2020;70(695):270-1.

3. Sommerhalder $\mathrm{K}$ et al. Internet information and medical consultations: experiences from patients' and physicians' perspectives. Patient Educ Couns. 2009;77(2):266-71.

4. National Information Board (NIB) Health secretary outlines vision for use of technology across NHS 2015. Available at: https://www. gov.uk/government/news/healthsecretary-outlines-vision-for-useof-technology-across-nhs. Last accessed: 13 November 2020.

5. The Phoenix Partnership (TPP). Homepage. 2020. Available at: https://www.tpp-uk.com. Last accessed: 13 October 2020

6. The National Health Service (NHS). The NHS App. 2020. Available at: https://www.nhs.uk/using-the-nhs/ nhs-services/the-nhs-app/. Last accessed: 13 November 2020.

7. Brant $\mathrm{H}$ et al. Using alternatives to face-to-face consultations: a survey of prevalence and attitudes in general practice. $\mathrm{Br} J$ Gen Pract. 2016;66(648):e460-6.

8. Donaghy $\mathrm{E}$ et al. Acceptability, benefits, and challenges of video consulting: a qualitative study in primary care. $\mathrm{Br} \mathrm{J}$ Gen Pract. 2019;69(686):e586-94.

9. Gov.uk. Guidance on shielding and protecting people defined on medical grounds as extremely vulnerable from COVID-19. 2020. Available at: www.gov.uk/government/ publications/guidance-on-shieldingand-protecting-extremelyvulnerable-persons-from-covid-19/ guidance-on-shielding-andprotecting-extremely-vulnerablepersons-from-covid-19. Last accessed: 13 November 2020

10. Tourangeau R et al. Web surveys by smartphone and tablets: effects on survey responses. Public Opin $Q$. 2017;81(4):896-929.

11. Wang $Y$ et al Do men consult less than women? An analysis of routinely collected UK general practice data. BMJ Open. 2013;3:e003320.

12. Mangin D et al. Multimorbidity, eHealth and implications for equity: a cross-sectional survey of patient perspectives on eHealth. BMJ Open. 2019;9(2):e023731.

13. Jones D et al. Impact of the COVID-19 pandemic on the symptomatic diagnosis of cancer: the view from primary care. Lancet Oncol. 2020:21(6):748.
14. Gray DM et al. Strategies for digital care of vulnerable patients in a COVID-19 world-keeping in touch. JAMA Health Forum, 2020; 1(6):e200734.

15. Casey $\mathrm{M}$ et al. Experiences with online consultation systems in primary care: case study of one early adopter site. Br J Gen Pract. 2017;67(664):e73643.

16. Helsper $\mathrm{CW}$ et al. Cancer has not gone away: a primary care perspective to support a balanced approach for timely cancer diagnosis during COVID-19. Eur J Cancer Care. 2020;29(5):e13290.

17. Symonds $\mathrm{R}$ et al. Patient perception of remote, digital NHS healthcare during the COVID-19 pandemic: a UK-wide survey. J Med Internet Res. 2020;DOI:10.2196/preprints.22629.

18. Wolf MS et al. Awareness, attitudes, and actions related to COVID-19 among adults with chronic conditions at the onset of the US outbreak: a cross-sectional survey. Ann Intern Med. 2020;173(2):100-9.

19. Goodyear-Smith F et al. Relationship between the perceived strength of countries' primary care system and COVID-19 mortality: an international survey study. BJGP open. 2020;4(4):DOI: 10.3399/ bjgpopen20X101129. 